\title{
Mixité et égalité dans la famille et à l'école
}

\section{Yveline Fumat}

\section{CpenEdition}

Journals

Édition électronique

URL : http://journals.openedition.org/trema/1069

DOI : 10.4000/trema.1069

ISSN : 2107-0997

\section{Éditeur}

Faculté d'Éducation de l'université de Montpellier

\section{Édition imprimée}

Date de publication : 1 juin 2010

Pagination : $7-20$

ISSN : 1167-315X

\section{Référence électronique}

Yveline Fumat, « Mixité et égalité dans la famille et à l'école», Tréma [En ligne], 32 | 2010, mis en ligne

le 01 juin 2012, consulté le 30 avril 2019. URL : http://journals.openedition.org/trema/1069 ; DOI :

10.4000/trema.1069

Ce document a été généré automatiquement le 30 avril 2019.

Trema 


\title{
Mixité et égalité dans la famille et à l'école
}

\author{
Yveline Fumat
}

on parle de mixité, on confond souvent plusieurs phénomènes qui ont été mêlés historiquement, et qui pourtant sont malgré tout distincts: la cohabitation de filles et de garçons dans les mêmes classes ou les mêmes établissements, la similitude des cursus d'enseignement, et l'éducation aux rapports de sexe.

Quand on pense à la mixité, on imagine des garçons et des filles partageant le même espace - dans la classe et à la récréation écoutant les mêmes cours, soumis aux mêmes règles de droit.

3 Mais le simple fait que les filles et les garçons cohabitent sur les bancs de l'école n'est pas en soi une valeur, une garantie d'égalité. Le premier et le meilleur démenti à cette croyance est bien l'exemple de la famille: c'est le lieu où cohabitent étroitement filles et garçons et pourtant l'éducation dans la famille, depuis toujours et encore maintenant, suppose une éducation aux rôles sociaux très fortement différenciée.

4 La victoire essentielle des filles a été le rapprochement des contenus de leur enseignement et l'ouverture des filières jusque là réservées aux garçons. C'est surtout parce que les filles ont pu faire des études, et parce que ces études ont été les mêmes que celles des garçons, que l'on s'est rapproché de l'égalité.

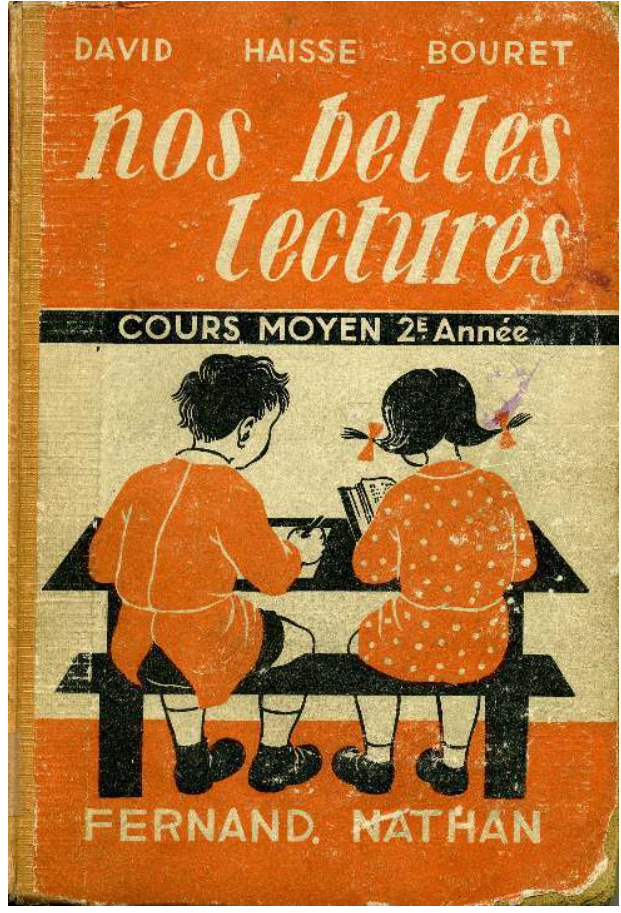


Dans l'idée de mixité on mélange donc plus ou moins la proximité physique et concrète des individus et la possibilité enfin donnée aux filles d'entrer dans des écoles ou des filières réservées jusque là aux garçons. C'est pourquoi la «mixité » est pensée comme une valeur, et se relie à l'idée d'un progrès vers l'égalité. Du même coup elle peut paraître suffisante pour réduire toutes les injustices. On dira : maintenant que toutes les études sont possibles pour les filles, que toutes les écoles, tous les cursus, et toutes les carrières leur sont ouverts, que demander de plus?

6 Il est certain que la situation des filles est sans commune mesure avec celle d'il y a cent ans où les possibilités d'études étaient encore très différentes : c'est seulement en 1919 qu'on crée un baccalauréat féminin. Et même sans commune mesure avec celle d'il y a trente ans, où peu de femmes encore entraient dans des carrières jugées masculines (chirurgien, policier, magistrat même, alors que dans ce métier elles sont maintenant plus nombreuses que les hommes). Est-elle pour autant complètement et définitivement transformée ? Ne reste-t-il pas des phénomènes curieux, comme celui des « choix que les filles ne font pas ", celui des filières scientifiques et techniques, puis des métiers qui en sont le prolongement? Ce qui donne à penser que des résistances inconscientes subsistent : sous- jacentes au "libre choix », des déterminations plus puissantes freinent encore les aspirations féminines. Actuellement on doit constater que la complète identité des cursus et la loi qui donne des droits égaux ne sont pas encore suffisants pour conduire à l'égalité réelle. Avant même l'entrée à l'école, l'éducation précoce suggère déjà aux filles et aux garçons des avenirs dissemblables, dessine des éventails de choix qui ne sont pas vraiment identiques, leur ouvre encore des «destinées» différentes. Il ne suffit pas de mettre en place des procédures d'orientation au collège, ou de faire des campagnes d'incitation, si plus profondément une "orientation» dans l'espace polarisée dedans/ dehors, ou un imaginaire plus limité, ont été construits précocement dans la famille. Ou encore si les vœux, les rêves des parents, les jeux et les jouets, ont favorisé chez l'enfant des dispositions différentes, et même des « schémas » de pensée distincts.

\section{L'éducation mixte et différenciée dans la famille}

7 Alors même qu'ils vivent ensemble, filles et garçons ne sont pas élevés de la même manière.

Avant même l'éducation par la parole, la première socialisation se fait en modelant directement les corps (posture, vêtements), en guidant les mouvements par le biais d'espaces structurés et d'objets spécifiques, en insérant l'enfant dans un monde polarisé selon les sexes. Dés la naissance une nette différenciation peut être provoquée par l'environnement physique (chambre, jouets), par la manière de s'occuper du bébé (de le porter, de le tenir, de le nourrir, de le stimuler, de le provoquer dans les jeux). On devient fille ou garçon à partir d'une base biologique certes, mais si elle est confirmée par les parents, par leurs attentes, leurs représentations, leurs attitudes, leur acceptation de ce sexe biologique. C'est seulement en comprenant l'ancienneté et la force des habitudes passives, par contacts corporels, fréquence des impressions reçues, que l'on peut imaginer toute la zone de non-dit où se construit et se différencie une fille ou un garçon. Les premières recherches de BELOTTI (1973) sur la manière dont on nourrit le bébé ont sans doute été complétées, nuancées depuis, et les comportements ont pu évoluer; elle a malgré tout inauguré un type de recherches qui a fait prendre conscience de la profondeur des déterminations premières. Certes la différenciation dans nos sociétés est 
sans doute moins forte qu'auparavant, mais on peut constater la rémanence de certaines attitudes : des études récentes montrent que la façon de nourrir et de jouer des pères reste bien différente : avec leur bébé garçon ils ont plus de contacts et d'interactions motrices (ils le bousculent, ils cherchent la bagarre) et avec les filles ils entrent en « conversation » en suscitant vocalisation et sourires.

Une grande part de l'éducation se fait en deçà du discours, par les échanges émotionnels qui valorisent ou dévalorisent les comportements. Pour la différenciation des sexes les réactions aux coups, aux chutes qui sont des moments très intenses émotionnellement, sont sans doute discriminantes. Il fallait que les garçons soient très courageux (pour en faire des soldats "aguerris »), ce caractère était aussi un marqueur de leur différence avec les filles (il ne fallait pas qu'ils deviennent des «femmelettes»). L'apprentissage de la marche, occasion inévitable de se faire mal, suscite des réponses diverses des adultes: l'émotion manifestée, l'exclamation de frayeur, donne sens à la douleur physique de l'enfant. Tout fait de ce moment capital un temps fort qui éduque l'enfant de manière particulière : la douleur est comme travaillée par l'entourage qui l'individualise, l'isole ou la néglige, la souligne ou la dénie ... L'anxiété personnelle du parent est sans doute déterminante ; mais elle est aussi très liée à l'époque, à la culture, et au sexe : il est certain que les garçons perdent là leur droit de pleurer, de manifester leur peur. Les filles au contraire, imaginées déjà comme plus fragiles, peuvent se plaindre, on accepte mieux qu'elles demandent à être consolées.

10 L'angoisse manifestée quand l'enfant s'éloigne donne déjà un style aux permissions qui viendront plus tard. Quand les parents ont intériorisé les rôles masculins et féminins traditionnels, encore maintenant, ils seront plus protecteurs pour la fille, et craindront qu'elle « aille trop loin» (ce qui renvoie à la fois au rapport à l'espace, à une certaine audace dans les initiatives, et à un éventuel écart au plan sexuel); dans le même temps, paradoxalement, la séduction, la coquetterie, sera plus encouragée chez la fille alors que l'on redoutera toujours les goûts « efféminés » chez le garçon.

11 Le discours parental - et il faut inclure dans ce discours tous les silences, les consentements implicites - signifient à l'enfant, au travers de chaque micro intervention, qu'il doit devenir fille ou garçon. Tous les interdits, mais aussi tous les encouragements, tous les éloges, qu'ils soient explicites ou «insinués » (BOURDIEU, 1972) contribuent à tracer pour l'enfant une aire d'action qui sera celle où il devra jouer son rôle. Par des mises en garde, des mises en demeure, des appels au dépassement, la famille instaure les prérogatives du garçon, les limitations de mouvement de la fille, l'éventail de choix des métiers souhaités, elle donne la notion de la valeur respective de chaque sexe, et l'exemple du respect ou du mépris dans les rapports entre les sexes. Certes les choix se sont élargis pour les filles, le choix n'est plus entre le mariage ou le couvent. Mais précisément il faut comprendre que les représentations conscientes sont parfois contredites par des attitudes ancestrales, qui perpétuent une façon d'élever les filles « à l'ancienne »; le rapport à l'espace, si profondément ancré, est sans doute encore transmis. À la fille le "dedans", les espaces clos, la maison, le ménage, le soin des enfants. Au garçon la recherche de l'aventure, de l'Espace, la maîtrise du monde par des machines, les combats avec les monstres des catalogues de jouets; les tenues de cosmonautes restent masculines. La nécessité de la séduction (pour trouver un géniteur et faire des enfants) reste l'apanage des filles. Certes l'imaginaire de la fille n'est plus bridé comme auparavant; on ne verra sans doute plus beaucoup de familles où l'on demande à la fille de se dévouer, de se sacrifier même, pour ses frères. Mais peut-on dire 
que l'on souhaite autant d'audace, d'initiative, d'esprit d'aventure pour la fille que pour le garçon? Peut-on trouver beaucoup de parents qui accepteraient de grand cœur que leur fille ne veuille pas d'enfant?

12 Cette assignation profonde et tacite à la perpétuation de la vie, reste plus forte pour la fille que pour le garçon. Que la fille se sente toujours sollicitée pour ce rôle de mère, qu'elle s'imagine devant plus tard concilier son métier et sa vie de famille, on le voit bien dans l'extraordinaire différence relevée par BAUDELOT et ESTABLET dans Allez les filles! (1992). Une des questions porte sur les critères de choix d'une profession: alors que le « temps libre » est mis en avant par $72 \%$ des filles, il n'est mis en avant que par $11 \%$ des garçons. Il est certain que l'on découvre là "en creux » une intériorisation du rôle familial anticipé très lucidement par les filles. Elles imaginent bien que seul ce temps libre leur permettra de concilier métier et activités à la maison, alors que les garçons n'y pensent même pas (dans leur livre plus récent Quoi de neuf pour les filles? les auteurs ne reprennent pas ce questionnaire, il aurait été intéressant de voir si cette réponse s'était modifiée). Ainsi les filles préfèrent, plutôt que des carrières prestigieuses mais trop prenantes, des professions plus "féminines », aux horaires plus souples, et souvent plus sédentaires, ce qui leur laissera du temps pour les enfants. Ce " choix » reflète bien, en creux, la division du travail domestique actuelle. Dans un documentaire récent sur les femmes, les projets d'avenir professionnels des jeunes filles gardaient cette même ambition "nuancée », le rêve corrigé aussitôt par une prise en compte de la maternité : «Oui je voudrais faire des explorations scientifiques ... mais comme je voudrais aussi trois enfants...enfin on verra bien ... ». Et une autre : «J'aimerais travailler dans le commerce international ... mais sans trop voyager quand même, en restant en France ».

\section{La mixité dans les institutions éducatives}

\section{1. Quand la ségrégation va de soi}

Il faut d'abord rappeler que pendant longtemps l'école n'était pas mixte, tout simplement parce que les filles n'étaient pas scolarisées; l'éducation exclusivement familiale des filles, pendant des siècles, a fait que le problème ne se posait même pas. Toute l'évolution étudiée par Philippe ARIES (1975) concernant le "sentiment de l'enfance ", la perception nouvelle de sa spécificité, sera liée à l'entrée dans les collèges des garçons uniquement. La nouveauté viendra de la mise à part des jeunes garçons qui seront désormais scolarisés, ce qui détachera le monde de l'enfance de celui de l'adulte, rendra la coupure plus visible. Rien de tel pour les filles - on oublie souvent de le souligner - qui recevront la même éducation familiale au contact des femmes, de leur mère surtout qui leur donnera l'exemple de tous les comportements féminins, ménagers, religieux, moraux ... La preuve en est bien cette identité persistante des formes vestimentaires alors que les garçons porteront des vêtements spécifiques, la « livrée de l'enfance ». Pour les filles «après le démailloter elles seront vêtues comme de petites femmes". Ariès dit bien que "sans scolarité propre les filles étaient très tôt confondues avec les femmes ». Il reconnaît que « le sentiment de l'enfance s'est éveillé d'abord au profit des garçons tandis que les filles persistèrent longtemps dans le mode de vie traditionnel ».

Quand les filles vont à l'école sous l'Ancien Régime, il s'agit surtout des "petites écoles » au niveau primaire, où elles apprennent à lire, moins souvent à écrire (car cela se faisait successivement et elles n'y allaient pas longtemps). Ni mixité, ni programmes identiques ; 
les écoles de garçons sont tenues par des prêtres, les écoles de filles par des religieuses. "Des enfants séparés, garçons d'un côté, filles de l'autre. C'est plus qu'une règle, une obsession. Dans les statuts synodaux, la recommandation est constante» (FURET et OZOUF, 1977).

L'enfant doit être préservé non seulement de toute occasion de contact sexuel, mais de «toute éventuelle pensée d'impureté sexuelle ». Il est vrai que l'insistance à réaffirmer la norme montre qu'elle était parfois transgressée. Il faut donc rappeler que la non mixité est d'abord l'effet d'une ségrégation des sexes voulue par la religion, et que les programmes différents sont également profondément liés à une vision de la Femme chrétienne, de sa Nature, de ses devoirs, de sa destinée familiale, qui explique bien le sens et la limitation du curriculum : les ordonnances épiscopales insistent sur la lecture - pour lire le catéchisme - sur l'apprentissage ménager et la couture, l'accent est mis sur la pédagogie des vertus chrétiennes, sur l'horreur des péchés, particulièrement ceux qui sont opposés à la pureté. L'inégalité qui apparait dans le curriculum, selon FURET et OZOUF, ne traduit pas d'abord une condamnation intellectuelle des femmes, mais une volonté acharnée de ségrégation des sexes : «C'est le plaisir qui est interdit non pas l'instruction".

On peut dire aussi que l'obsession de la "pureté », de la virginité, de la fidélité, venant du modèle féminin chrétien, font que l'instruction est du même coup complètement subordonnée à l'éducation, et limitée dans ses objectifs et ses matières. Les filles n'ont pas besoin de savoir, à la fois parce qu'elles doivent avant tout devenir mères, et doivent limiter leur curiosité et leurs intérêts pour le «dehors». Dans toutes les histoires racontées aux jeunes filles, dans la littérature de loisir, sont réprimées, non seulement la sexualité, mais bien de proche en proche la sensualité, et tout désir : la petite fille qui court trop, qui a soif, boit trop avidement, et tombe malade pour ces « débordements " ... au contraire de la jeune fille modeste, occupée aux travaux d'aiguille, qui reste assise sagement à la fenêtre. Des auteurs comme Laurent de Jussieu (Histoires et causeries à l'usage des jeunes filles chrétiennes) n'hésitent pas à donner la taupe comme modèle de vie obscure et discrète (FUMAT, 1980).

\section{2. La mixité et l'égalité dans l'école républicaine}

17 Que devient la mixité lorsque la République proclame l'enseignement obligatoire, gratuit et laïc en 1881? La troisième République, avec Jules FERRY et Ferdinand BUISSON, reprend les idées des révolutionnaires de 1789 (CONDORCET, LAKANAL) et affirme un droit à l'instruction pour tous, y compris les filles, elle construit des écoles primaires dans les villages les plus reculés. En 1879 elle crée une école normale de filles dans chaque département. Elle achève ainsi un mouvement d'alphabétisation commencé avant elle; mais elle scolarise ainsi toutes les filles et on n'a peut être pas assez souligné l'importance de ce phénomène non seulement au plan du retard comblé pour les filles, mais au plan de l'éducation des garçons: l'écart va se réduire entre la culture familiale, jusque là purement traditionnelle, et transmise uniquement en langue régionale et la nouvelle culture scolaire, liée au français national, au progrès des sciences et des techniques, aux valeurs de la république. Les savoirs scolaires communs, donnés désormais aux filles les rapprochent de l'univers des garçons.

Pour le niveau primaire le curriculum devient pratiquement identique, sauf pour la couture, les travaux manuels, la gymnastique; mais la ségrégation physique est 
maintenue. La troisième république construit des écoles primaires pour les filles et les garçons mais sous le même fronton - où souvent est inscrite la devise Liberté Egalité Fraternité - les bâtiments symétriques accueillent d'un côté les filles, de l'autre les garçons. Ainsi la ségrégation des sexes est-elle à la fois corrigée et maintenue. Corrigée car les cursus se rapprochent et les écoles mêmes sont contiguës. Maintenue car la différence de traitement semble encore naturelle; les regroupements sont occasionnels (une chorale, une fête où l'on a besoin pour une pièce de rôles masculins ou féminins). Si les classes uniques à la campagne accueillent ensemble filles et garçons, cela semble bien une mesure économique, un pis aller, une exception par rapport à une norme implicite qui reste non discutée.

19 Pour l'enseignement secondaire, l'identité de cursus sera bien plus longue à obtenir et les résistances à l'élévation du niveau d'instruction des filles seront fortes pendant tout le XIXe siècle. Sans retracer toutes les étapes de la marche vers l'égalité, choisissons de revenir sur les différents arguments qui ont été développés au moment du vote de la loi Camille SÉE qui a vu la création de Lycées de Jeunes filles en 1880. Jusque-là il n'y avait pas d'enseignement public structuré pour les jeunes filles, seulement l'enseignement donné par les congréganistes, mais aussi un enseignement donné dans divers cours privés, souvent hebdomadaires et tenus par des hommes, où les jeunes filles assistaient avec leur mère assise au fond de la classe, celle-ci servant de répétitrice dans la semaine. Les « cours secondaires » de Victor DURUY en 1867 furent une expérience intéressante, mais de courte durée, qui a néanmoins servi de premier modèle à l'enseignement public.

En 1880 parmi toute la presse, (analysée par Mona OZOUF, 1982) bien sûr les journaux conservateurs vont défendre un statu quo qui donnait aux congrégations le quasi monopole de l'éducation féminine: ils dénoncent l'entreprise de "déchristianisation » des républicains. Certains comme Le Figaro se contentent de ridiculiser le futur « lycéen en jupons » ou encore donnent un argument - massue en faisant remarquer que la langue française elle-même proteste contre la nouvelle loi puisque on ne peut pas dire "des docteuses, des professeuses ». Mais d'autres journaux vont plus loin dans l'analyse des motifs et des effets de cette nouvelle législation.

21 La Civilisation affirme le 24 novembre 1880 : «En vérité ce dont il s'agit c'est de soustraire la Femme à l'influence du christianisme : on espère y arriver en mettant dans l'éducation des filles les enseignements d'une science vaine et superficielle à la place des préceptes divins. » On reproche aux républicains de substituer aux sentiments élevés, aux émotions pieuses, l'étude de l'hygiène de la première enfance, l'allaitement étranger et artificiel ... " une éducation toute darwinienne et scientifique (...) et de faire ainsi des athées et des positivistes ». C'est Le Gaulois (25 novembre 1880) qui annonce dramatiquement : «! ... La jeune fille avait été préservée de l'éducation garçonnière et des brutalités de la science... cette paix candide de la jeune fille, cette délicieuse floraison de pudiques désirs, ces élans d'idéale bonté qui plus tard font l'amour de l'épouse, et le sacrifice de la mère (...) tout cela va disparaître. On va supprimer la jeune fille ! (...) On leur apprendra tout, même la rébellion contre la famille, même l'impureté (...), elles n'auront même pas été vierges avant de devenir femmes, et quand elles auront conquis les grades universitaires, elles se donneront, comme les élèves du conservatoire, au premier homme qui passera ... ». On voit de manière lumineuse dans ce texte ce qui est redouté : l'effet de l'instruction sur l'image et le rôle sexuel de la femme : l'idéal de la jeune fille pure, dévouée, assignée à son rôle d'épouse et de mère se détache avec force; ce que les nouveaux savoirs scientifiques 
viennent troubler, avec «brutalité », violer pour ainsi dire, c'est la jeune fille de toujours, risquant d'en faire une rebelle, une volage, voire une putain ...

Mais les articles des journaux républicains ne sont pas moins intéressants car on voit d'abord que l'élévation de l'instruction des filles n'est nullement souhaitée pour les filles elles-mêmes, pour leur émancipation, leur bonheur, leur développement personnel ... mais pour des raisons politiques et une meilleure harmonie de la famille. Il faut que la femme ne soit plus "sur les genoux de l'Église », et que cesse le divorce à l'intérieur même des familles entre l'Homme et la Femme. Dans la présentation de la nouvelle loi à la chambre, Camille SÉE lui-même déplore qu'actuellement à la maison la Mère parle à la jeune fille le langage de la superstition, et le Père celui de la Raison (...) Appelée à vivre en communion de sentiments et d'idées avec un mari, appelée à élever ses enfants, elle a droit à une instruction qui soit digne d'elle ». Il déplore la nullité de l'enseignement dans les couvents et leur manière d'oublier le siècle: «La France n'est pas un couvent, la femme n'est pas dans ce monde pour être religieuse ; elle est née pour être épouse, elle est née pour être mère ».

Même si les journaux républicains s'accordent sur le principe de la loi, les controverses resteront très vives sur les programmes et surtout les internats. Beaucoup doutent encore de la capacité de la jeune fille à aborder les notions scientifiques et demanderont une "pédagogie spécifique». Les internats surtout divisent les esprits et les républicains rejoignent parfois les journaux les plus conservateurs. Le journal Le Voltaire, qui a pourtant plutôt soutenu la loi Camille SÉE, publie ensuite un article intitulé «la fille homme » pour s'élever contre les internats, qu'il ressent comme un « casernement ».

Le point de vue des journaux d'extrême gauche est le plus curieux (La Citoyenne 5 juillet 1882). Ils sont pour la création des lycées destinés aux filles de la bourgeoisie; mais ils donnent deux types d'arguments qui laissent perplexes. Le premier: "Comprenez bien qu'il faut des femmes instruites pour instruire vos filles, des femmes instruites pour entrer dans les professions libérales comme la médecine, afin de ménager la pudeur de vos femmes et de vos filles ». Ainsi par un détour subtil, on retombe sur le sexe, toujours le sexe ... Même chez ceux qui sont à l'avant - garde politique, la jeune fille et la Femme gardent un rôle inchangé.

Le deuxième argument est encore plus curieux à nos yeux : « Femmes du peuple, il est de votre intérêt que les filles de la bourgeoisie s'instruisent, car si elles n'ont pas le savoir, la possibilité de disputer aux hommes leurs emplois, elles vous disputeront à vous l'aiguille, (...) et renforceront les rangs des couturières et des lingères, et par la concurrence feront baisser vos salaires ... ». On voit que pour l'ultra gauche le rapport de classe est seul déterminant ; les rapports de sexe et la domination masculine sont totalement invisibles.

Tout cet argumentaire révèle bien la force des stéréotypes : à une certaine époque ils sont vraiment « indépassables » pour tous. Même si certains pressentent que l'instruction des filles est un obscur danger pour le modèle familial, personne ne peut vraiment imaginer que cette instruction et le développement du travail féminin bouleverseront totalement la société au siècle suivant. Car, il faut le souligner, ce n'est pas tant l'élévation des connaissances chez les filles qui sera capital, mais le fait que cette instruction accrue sera convertie en métier féminin. 


\section{3. Comment progresse la mixité au XXe siècle?}

27 Les filles ont droit maintenant à un enseignement secondaire mais au départ les programmes ne sont pas les mêmes que ceux des garçons : elles ont un enseignement sans langues anciennes ce qui les défavorise pour bon nombre de professions libérales et surtout un simple « certificat de fin d'études secondaires ». Le vrai baccalauréat, qui leur permet d'entrer en faculté, ne viendra qu'en 1919. C'est en 1924 que seront unifiés complètement les programmes. Du coup le corps enseignant peut être mixte dans les lycées.

Les écoles normales restent distinctes mais leurs programmes sont pratiquement identiques avec cependant des cours d'» agriculture » pour les garçons (qui sont censés éclairer les paysans) et un cours de "couture » et d'» économie domestique » pour les filles (où l'on apprend à enlever les taches et à donner des conseils d'allaitement). Encore en 1960 filles et garçons vivent leur vie de normaliens dans deux établissements séparés avec des rencontres épisodiques: la chorale, la pièce de théâtre. Mais des rites de bizutage, créés par les élèves et tolérés par la hiérarchie, tissent des liens souterrains : il est d'usage que les élèves reçus avec le même rang au concours dans le même département, et appelés "mari » et «femme » pédagogiques, entrent en correspondance la première année : le «mari » doit envoyer une déclaration, souvent un poème d'amour, mais qui aura la particularité d'avoir été corrigé par le « père » voire le " grand-père » qui ont eu le même rang les années auparavant. Ainsi le beau poème d'amour se voit surligné, annoté, complété par des remarques obscènes et des dessins pornographiques.

Le nombre de mariages « normaliens » montre bien que la non mixité des écoles n'est pas un obstacle majeur. Les «bals normaliens» sont acceptés par l'institution. Il semble même que très consciemment le Directeur chez les garçons et la Directrice chez les filles pensent que le meilleur avenir pour les élèves - maitres est le poste double. ${ }^{1}$

\section{4. Comment a cessé la ségrégation physique dans les classes et les établissements?}

30 On peut dire que la ségrégation physique a cessé progressivement et sans bruit. Entre 1950 et 1970, selon les niveaux d'enseignement, les lieux géographiques - villes ou campagne - , les situations concrètes, la mixité se met en place ; il est frappant de voir que la mixité " physique » n'est l'objet d'aucun débat d'idées, d'aucune discussion nationale. La ségrégation était délibérée, argumentée. Alors que la mixité physique qui lui succède, ne paraît pas faire problème ni recevoir d'opposition forte. Elle semble moins liée à une organisation de l'institution scolaire, qu'à une simple conséquence de l'évolution globale des mœurs.

31 Deux décrets marquent un constat de fait, prennent acte d'une lente évolution : 1963, décret qui institue la mixité comme régime normal dans les CES, et en 1975 la Loi HABY du 11 juillet relative à l'éducation « rend obligatoire la mixité dans tous les établissements publics ».

32 C'est justement «l'achèvement » en quelque sorte de la mixité au plan du droit aux mêmes études, et de la cohabitation sur les mêmes bancs, qui permet d'approfondir la réflexion et de poser de manière plus complexe le problème de l'égalité. Aussi bien des mouvements féministes militants à partir des années 1970, que des sociologues ou 
psychologues qui prennent en compte la variable «sexe", vont montrer que les filles ne semblent pas se saisir de toutes les possibilités nouvelles qui leur sont offertes. On constate que les filles ont toujours moins d'ambition ou de confiance en elles, et qu'elles hésitent à s'engager dans certaines filières :

- elles désertent certaines filières techniques,

- elles ne choisissent pas de continuer en filière scientifique même si leurs résultats sont bons,

- elles ne « convertissent » pas forcément leur bac scientifique en orientation scientifique,

- elles ne se voient pas dans certaines carrières où conduisent pourtant leurs études scientifiques,

- si elles pensent aux filières scientifiques, il s'agira plutôt de la biologie (plus près de la vie),

À chaque carrefour d'orientation, apparaît une méfiance vis-à-vis de la Science qu'a plus particulièrement étudiée Marie DURU-BELLAT. (1990). Dès 6 ans les filles considèrent que la science et la technique sont des domaines "masculins ", des domaines où règnent la compétition, et la seule rationalité (et dans leurs jeux les filles n'aiment pas trop la «bagarre »). La science est froide alors que tout ce qui touche à l'humain leur semble plus intéressant (travail social psychologie). C'est sans doute aussi l'imaginaire exploratoire qui est en cause (astronomie, physique, recherche en général). Ainsi il faudrait au moins donner une autre image de la science : trouver des exercices, des exemples d'application, qui parlent davantage aux filles.

Toujours d'après DURU-BELLAT, c'est l'achèvement de la mixité qui a fait prendre conscience de tout le poids des stéréotypes de sexes qui non seulement étaient antérieurs et extérieurs à l'école, mais étaient perpétués, reconduits, parfois même accentués par elle.

Les premières études ont porté sur les manuels scolaires (images des occupations des deux sexes toujours décalées) puis sur l'orientation des filles (filières, métiers). Sur les relations entre professeurs et élèves dans la classe : le traitement différent des filles et des garçons a été observé par vidéo, avec mesure des interactions ; les enseignants jouent des rivalités, s'appuient sur les stéréotypes, confient les tâches selon les stéréotypes; ils stimulent différemment garçons et filles, contrôlent plus les garçons dont ils redoutent plus l'indiscipline ... Des recherches sur l'évaluation ont été nombreuses : les garçons sont encouragés comme s'ils n'exploitaient pas toutes leurs possibilités... À résultat égal, par ailleurs, ils auront plus confiance en eux ... (DURU-BELLAT, 1990).

$\mathrm{Au}$ lieu de perpétuer, au travers d'une neutralité apparente, des inégalités profondes de sexe, l'école ne doit-elle pas essayer de les combattre ? En acceptant les rôles rigides entre les sexes, en reconduisant des divisions sociales considérées comme "naturelles", elle restait encore loin de ses principes fondamentaux. 


\section{La mixité considérée comme une véritable co- éducation}

37 Ainsi la mixité entendue comme identité de cursus, ouverture des filières, cohabitation dans les mêmes lieux, ne suffit pas à promouvoir l'égalité des filles.

Devant ce constat dans certains pays, on a remis en cause la mixité elle-même, mais cela à partir de points de vue qu'il faut malgré tout distinguer : certaines ont dénoncé la mixité comme "assimilation androcentrique implicite» et souhaité pour les filles un enseignement de filles ; à l'opposé des défenseurs des garçons, devant les succès scolaires féminins, commencent à penser que les garçons seraient mieux protégés dans des filières exclusivement masculines. Des deux côtés on semble prêt à reconstituer des ghettos, dans le même esprit que celui de la création d'écoles de Noirs; d'autres imaginent une séparation mais provisoire, pour certains cours seulement, des lieux qui permettraient aux filles d'être « discordantes, audacieuses, énergiques » (MOSCONI, 1998).

Dans l'esprit même de l'école républicaine, on peut estimer qu'il faut aller plus loin et arriver à une véritable co-éducation des filles et des garçons. Ce qui signifie qu'il faut :

- prendre conscience des stéréotypes et lutter contre leur reproduction,

- combattre les attitudes sexistes de mépris,

- travailler concrètement à instaurer des attitudes de respect de l'autre.

Mais du même coup l'éducation aux rapports de sexe va s'instaurer dans une zone qui dépasse aussi bien la simple " éducation sexuelle » qui se faisait sur un plan uniquement physiologique (anatomie et mécanisme de la reproduction) que la simple "éducation civique » qui s'adressait à des êtres neutres, asexués.

La recherche de l'égalité signifie que l'on met en avant le respect de la personne humaine : C'est une attitude éthique que l'école doit construire et transmettre si elle veut être fidèle jusqu'au bout à ses idéaux. La persistance d'une hiérarchie entre les sexes héritée de la société traditionnelle patriarcale et d'une certaine interprétation des religions (catholicisme, judaïsme, islam) n'a pas sa place à l'école de la République. Non seulement celle-ci ne doit pas la relayer, mais elle doit la combattre.

Il faut donc travailler consciemment en éducation civique par exemple les thèmes de l'égalité entre filles et garçons, et du respect mutuel.

Des directives ont été données en 2000 (BO du 24-10) par le ministère de l'Éducation nationale et un décret publié sous la signature de Jack LANG (d'après les travaux d'une commission inspirée par des chercheuses en sciences de l'éducation ) fournissant des exemples très précis d'activités en classe visant à combattre les stéréotypes, «De la mixité à l'égalité ", porte sur les interactions dans la classe, les activités de groupe, l'éducation à l'orientation, l'éducation à la santé et la prévention des violences sexuelles et sexistes. Un exemple pris dans le scénario 6.5 : «À la récréation les filles n'osent plus traverser la cour du collège, parce qu'un groupe de garçons les interpelle à propos de leur tenue en proférant des propos sexistes... Recommandation: intervenir pour mener un travail de réflexion avec les protagonistes, faire découvrir la part de conformisme social de leur comportement; travailler sur les représentations idéalisées de l'Homme et de la Femme que véhiculent les médias; faire prendre conscience que les propos sexistes instaurent un rapport de force qui peut conduire à terme à d'autres formes plus violentes ». Le document se veut un outil très concret pour « libérer nos sociétés de l'un 
de ses carcans les plus archaïques et pour parvenir à une parfaite égalité de condition entre les hommes et les femmes ».

Le problème de l'égalité a recoupé celui de la laïcité lorsque s'est posé le problème du foulard islamique sur lequel la loi du 15 mars 2004 a finalement tranché après la grande enquête de la commission Stasi : dans l'espace scolaire elle demande aux jeunes de s'abstenir de tout signe religieux ostensible ... Le foulard islamique signe d'appartenance à l'Islam, a paru incompatible avec les Valeurs de la République dans la mesure où il était aussi un signe de la "non égalité » des femmes, un signe de leur soumission (le voile rend visible «l'enclos invisible où elles sont maintenues » selon BOURDIEU (1998).

La situation scolaire actuelle où des élèves sont d'origine maghrébine accentue le problème : en arrivant porteurs des valeurs familiales d'une autre culture, ils posent de manière plus aiguë encore le problème de l'égalité: des discussions sur les discriminations sexistes ou racistes, sur la tenue des filles, leur droit à la «jupe » mais aussi au mariage «choisi », sont nécessaires. L'école doit défendre l'égalité, refuser les injustices non seulement à l'école, mais aussi à l'extérieur.

Le BO du 1er février 2007 n'estime pas que le problème de l'égalité soit résolu ; c'est une convention entre de nombreux ministères qui affirme ceci en introduction:

«Aujourd'hui les femmes poursuivent des scolarités jusqu'aux plus hauts niveaux de formation : elles représentent plus de la moitié de la population active et accèdent à des métiers et à des niveaux hiérarchiques longtemps réservés aux hommes.

8 Toutefois force est de constater la persistance de difficultés rencontrées par les femmes dans leur trajectoire professionnelle : elles sont plus souvent que les hommes confrontées au chômage, aux emplois précaires, au temps partiel contraint et souvent moins bien rémunérées ».

Le document constate la réussite scolaire des filles (en terme de durée moyenne des études, de niveau moyen des diplômes, de taux de réussite aux examens) mais toujours leur sous - représentation dans les filières les plus prestigieuses et les plus porteuses d'emplois, (le document constate un certain progrès accompli dans les écoles d'ingénieurs : la proportion de femmes passe de $15,7 \%$ à 24,7 ) et leur sur représentation dans les filières littéraires, les IUFM, les filières para médicales et sociales; les garçons sont plus nombreux dans les filières scientifiques et industrielles dans les IUT et les écoles d'ingénieurs. D'où la nécessité de " permettre aux filles et aux garçons de sortir de tout déterminisme sexué de l'orientation, pour laquelle les aspirations et les compétences doivent prévaloir.»

50 À cet effet des mesures seront prises (écarter tout stéréotype des brochures d'orientation, féminiser les noms de métier...).

51 Le souci de l'orientation semble premier, l'éducation plus en profondeur ne semble abordée que comme "levier activé en amont», condition préalable à une meilleure orientation professionnelle. Le document souligne bien la nécessité de «sensibiliser aux 
stéréotypes sexistes ", mais il ne développe pas autant que celui de 2000 les moyens pour le faire.

Par contre il précise plus nettement qu'il faut « assurer une information sur les violences spécifiques subies par les filles issues de l'immigration, tels que les mariages forcés ou les mutilations sexuelles ", et qu'il faut renforcer la lutte contre le harcèlement sexuel, et contre les formes de bizutage à caractère sexiste ou sexuel. Il souligne la nécessité de former à ces problèmes les membres du système éducatif.

La convention implique une formation à l'égalité de tous les membres du système éducatif (formation des formateurs) qui effectivement n'était pas très développée.

Ainsi la France, dans la logique de sa tradition républicaine, cherche plutôt à réaliser une mixité plus effective et plus profonde; elle ne semble pas remettre en question la cohabitation entre filles et garçons, comme dans d'autres pays où la question fait débat.

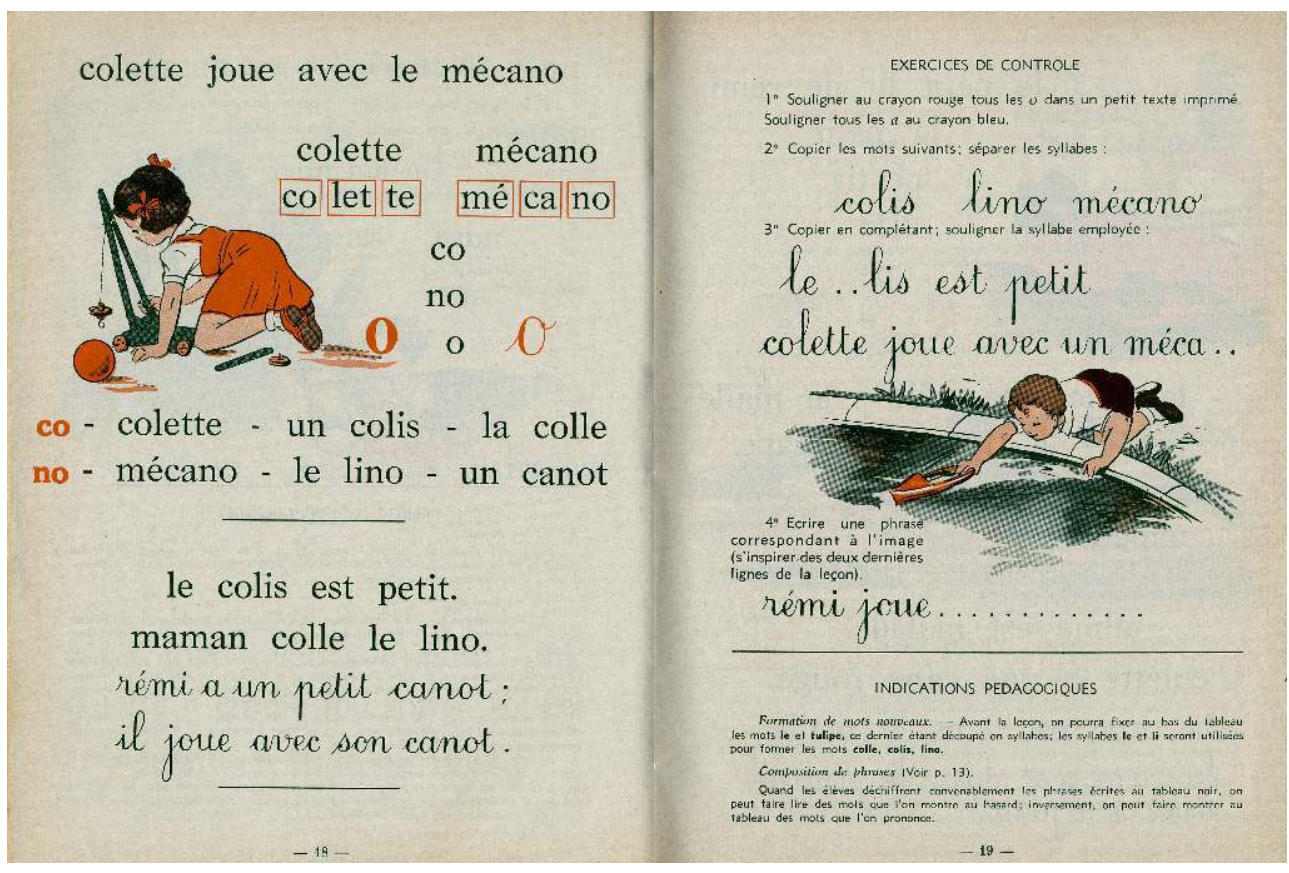

Faibles stéréotypes de genre. Image tirée de Rémi et Colette : méthode active de lecture ... $7^{\text {er }}$ [et $2^{\text {e }}$ livrets] / J. Juredieur ; E. Lourlevat ; illustrations de René Bresson.- Paris : Magnard, 1965.- 59 p. : ill. en coul. ; 22 cm. - pp. 18-19. Source : CEDRHE

\section{BIBLIOGRAPHIE}

ARIES, P. (1975). L'enfant et la vie familiale sous l'ancien régime. Paris : Points Histoire Seuil.

BAUDELOT, C. \& ESTABLET, R. (1992). Allez les filles! Paris : Seuil.

BAUDELOT, C. \& ESTABLET, R. (2007) Quoi de neuf chez les filles? Paris : Nathan.

BELOTTI, E. (1973). Du côté des petites filles. Paris : Édition des femmes. 
BOURDIEU, P. (1972). Esquisse d'une théorie de la pratique. Paris : Droz.

BOURDIEU, P. (1998). La domination masculine. Paris : Seuil.

DURU-BELLAT, M. (1990). L'École des Filles. Paris : L'Harmattan.

FUMAT, Y. (1980). La socialisation des filles au XIX ${ }^{\circ}$ siècle, Revue française de Pégagogie, $\mathrm{n}^{\circ} 52$.

Paris : INRP.

FUMAT, Y. \& Acherar, L. coord. (1994). Que veulent les filles ?, Les cahiers du CERFEE, $\mathrm{n}^{\circ} 14$.

Montpellier : Université Paul Valéry-Montpellier III.

FUMAT, Y. (1998). La socialisation primaire peut-elle être démocratique ? Les cahiers du CERFEE, $\mathrm{n}$

- 15 (TOZZI, M. coord.), p. 53-59. Montpellier : Université Paul VALERY-Montpellier III.

FUMAT, Y. \& PITHON, G. coord. (1994). Famille/École : quelles médiations ? Les cahiers du CERFEE, $\mathrm{n}^{\circ}$ 16. Montpellier : Université Paul Valéry-Montpellier III.

FURET, F. \& OZOUF, M. (1977). Lire et Écrire. Paris : Minuit.

GARNIER, Ch. (1995). Ce dont les enfants sont capables. Paris : Métailié.

KNIBIEHLER, Y. (1989). De la pucelle à la minette. Paris : Messidor.

LELIEVRE, C. \& LELIEVRE, F. (1991). Histoire de la scolarisation des filles. Paris : Nathan.

OZOUF, M. (1982). L'école l'église, la République. Paris : Cana.

OZOUF, M. (2009). La composition française. Paris : Gallimard.

MAYEUR, F. (1979). L'éducation des filles en France au XIX' siècle. Paris : Hachette.

MOSCONI, N. (1989). La mixité dans l'enseignement secondaire : un faux semblant ? Paris : PUF.

MOSCONI, N. (1998). Égalité des sexes en éducation et formation. Paris : PUF.

ZAIDMAN, C. (1996). La mixité à l'école primaire. Paris : L'Harmattan.

Décret 3 août 1963 sur la mixité des collèges d'enseignement secondaire.

Décrets d'application (28 décembre 1976) de la Loi HABY du 11 juillet 1975, décrets sur la mixité obligatoire dans l'enseignement primaire et secondaire.

B.O. du 24 octobre 2000, signé Jack LANG, « De la mixité à l'égalité », p. 1-21+ Nº 10, hors série.

B.O. $n^{\circ} 5$ du 1 février 2007, p. 254. «Convention pour l'égalité entre les filles et les garçons, les femmes et les hommes, dans le système éducatif ». Convention du 29-6-2006 (NOR :

MENE0603248X)

\section{NOTES}

1. Par exemple la Directrice de l'EN de Nice en 1956 dans son cours de " morale professionnelle » de 4ème année, étudiait très concrètement la situation de l'institutrice de village de montagne fraîchement nommée et nous mettait en garde contre des "faiblesses" liées à la solitude; elle affirmait que le mariage avec le collègue du village voisin était préférable à la mésalliance avec le cantonnier. Mais bien sûr elle n'allait pas jusqu'à nous mettre en garde au sujet des avances de Monsieur le Maire, voire de l'Inspecteur primaire. 


\section{RÉSUMÉS}

C'est la mixité des études qui a permis progressivement l'émancipation des filles et leur accès à tous les métiers. Mais la mixité comprise comme cohabitation dans les mêmes lieux et similitude des cursus ne suffit pas. Même si toutes les filières sont maintenant ouvertes aux filles, des résistances inconscientes subsistent - par exemple pour les choix d'orientation - qui viennent de la socialisation primaire par la famille. La mixité ne peut conduire à une véritable égalité que si l'école la transforme en co-éducation.

Mixed-sex education has progressively made the emancipation of girls possible; it has also allowed them to have access to any kind of job. Yet understanding mixed-sex education as being together in the same places and following the same curricula is not enough. Even though all courses are now open to girls, unconscious resistance persists - for instance in career path choices - stemming from primary socialization in the family. Mixed-sex education will only result in true equality if school turns it into genuine co-education.

\section{INDEX}

Keywords : co-education, equality, mixed sex education

Mots-clés : co-habitation, égalité, mixité

\section{AUTEUR}

\section{YVELINE FUMAT}

Professeur émérite Université Paul Valéry - Montpellier III 